\title{
Secondary Electron Contrast in STEM Electron Beam-Induced Current (EBIC): a Path Towards Mapping Electronic Structure
}

\author{
William A. Hubbard ${ }^{1}$, Matthew Mecklenburg ${ }^{2}$, Ho Leung Chan ${ }^{1}$, and B. C. Regan ${ }^{1}$ \\ 1. Department of Physics \& Astronomy and California NanoSystems Institute, University of California, \\ Los Angeles, USA. \\ 2. Center for Electron Microscopy and Microanalysis, University of Southern California, Los Angeles, \\ USA.
}

Image contrast in a given mode of electron microscopy is partially determined by the history of the electrons which are collected to form the image. In conventional (TEM) and scanning transmission electron microscopy (STEM), images are generated by collecting beam electrons after they pass through an electron-transparent sample. Detectors used in both scanning electron microscopes (SEM) and STEM, such as secondary (SE) and backscattered electron (BSE) detectors, form maps by associating the beam's position with its production of additional electrons. In traditional electron beam induced current (EBIC) images, current, plotted as a function of beam position, is generated by the separation of beam-induced electron-hole pairs by an electric field within the sample [1]. Here we report STEM EBIC contrast that maps hole current resulting from the emission of SE in electron-transparent devices and is entirely distinct from the contrast mechanisms mentioned above. Secondary electron emission EBIC, or SEEBIC, shows promise for STEM mapping of electronic properties, including connectivity, conductivity, and work function.

An electron beam incident on a sample will generate many SEs, leaving behind holes. In SEEBIC, these holes become a current, equal and opposite to the current of emitted SEs, that is measured by a transimpedance current amplifier (TIA) as the STEM beam scans the sample [2]. In our samples, the only path with significant conductance to ground goes through the TIA. The SEEBIC signals we observe are much smaller than typically reported EBIC signals caused by electron-hole pair separation (a few pA for a $100 \mathrm{pA}$ beam current incident on a metal electrode, compared to $\sim \mathrm{nA}$ for traditional EBIC [1]) and can be observed in the absence of applied or intrinsic electric fields.

Relative to STEM with off-sample SE detectors, STEM SEEBIC can provide a SE detection efficiency of unity, where even SEs emitted from the beam exit surface and buried interfaces are counted. Unlike a SE detector, SEEBIC can map SE emission independently in two electrically well-isolated conducting regions. In Fig. 1, a different EBIC signal was collected in each of the electrodes, as indicated by the blue and red current meter symbols in the right two images. When the beam is incident on an electrode connected to a TIA, SE emission, resulting in positive current in the TIA, produces bright contrast. Some $10 \%$ of the SE emitted from this electrode are recaptured by the opposing electrode, producing dark contrast in the other EBIC channel.

In the Fig. 1 images, a subtle step in the contrast appears between the electrodes running from top to bottom of each EBIC image. This step indicates a hole current boundary, on either side of which holes produced on the insulating membrane travel to ground preferentially via one of electrodes. At the top of the EBIC images, where the edges of the electrodes near this boundary are clean and straight, the line is situated halfway between the electrodes. Near the electrodes' closest approach, at the bottom of the images, poor liftoff has left metallic nanoparticles between the probes. This debris region provides a 
lower impedance path to the left electrode, perhaps due to percolative conductance, which moves the inter-electrode boundary towards the right electrode. Similar deployment of SEEBIC could be used, for example, to study the formation of conductive filaments in next-generation memory elements.

Applying a bias to an electrode will alter its SE emission properties and, consequently, the SEEBIC signal. A positively biased electrode will attract and re-capture some SE, decreasing the SE signal, while a negative bias may increase SE production in a high-, rather than ultra high-, vacuum environment [3]. We acquired SEEBIC image stacks of $\mathrm{Al}(100 \mathrm{~nm})$ and $\mathrm{Pt}(25 \mathrm{~nm}$ with a $5 \mathrm{~nm}$ Ti adhesion layer) electrodes under bias values ranging from $-9 \mathrm{~V}$ to $9 \mathrm{~V}$. Under positive bias the signal decreased $(\sim 60 \%$ of SE emission suppressed at $9 \mathrm{~V}$ ) as expected. However, under negative bias the SE emission increased, possibly due to our vacuum conditions as mentioned earlier. Using a model of the SE energy spectrum [1], we fit the SEEBIC as a function of positive bias [1] and obtain reasonable estimates of the work functions of Al and $\mathrm{Pt}(4.3 \pm 0.1 \mathrm{eV}$ and $5.6 \pm 0.4 \mathrm{eV}$, respectively)[4]. Successive measurements of the same regions, however, produce systematically larger work function estimates, possibly due to contamination.[5]

\section{References:}

[1] L Reimer in "Scanning electron microscopy: physics of image formation and microanalysis," (Springer, Berlin).

[2] ER White, A Kerelsky, WA Hubbard et al, Applied Physics Letters 107 (2015), 223104.

[3] W Chang et al, $28^{\text {th }}$ Aerospace Sciences Meeting and Exhibit Proceedings (2000).

[4] J. Hölzl in "Solid surface physics," (Springer, Berlin).

[5] This work has been supported by FAME, one of six centers of STARnet, a Semiconductor Research Corporation program sponsored by MARCO and DARPA, by National Science Foundation (NSF) award DMR-1611036, and by NSF STC award DMR-1548924. The authors acknowledge the use of instruments at the Electron Imaging Center for NanoMachines supported by NIH 1S10RR23057 and the CNSI at UCLA.
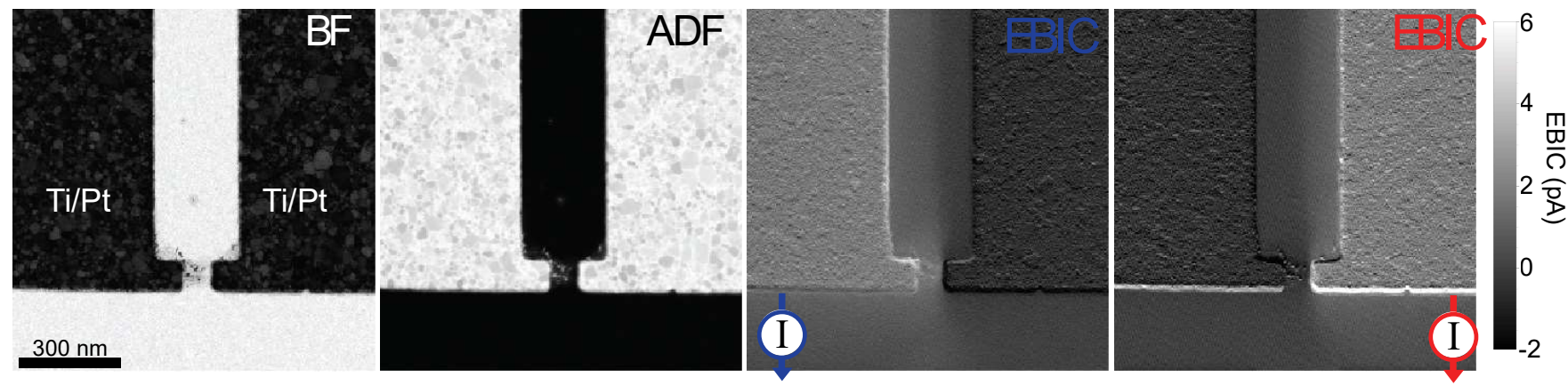

Figure 1. Conventional STEM, and STEM SEEBIC images of Ti/Pt electrodes. The simultaneouslyacquired bright field (BF), annular dark field (ADF), and STEM EBIC images show Ti/Pt (5/25 nm) electrodes patterned on top of $20 \mathrm{~nm}$ of $\mathrm{Al}_{2} \mathrm{O}_{3}$ on a $\mathrm{Si}_{3} \mathrm{~N}_{4}$ membrane. A blue or red symbol indicates the electrode connected to the TIA in each STEM EBIC image. While the BF and ADF images show this residue, they do not show to which of the two electrodes a given patch of residue is best connected. The current scale at the lower right applies to both EBIC images. 